\title{
Le stockfisch et la morue mis en recette
}

Manger du poisson sur tout le territoire français depuis le Moyen Âge

\section{Gérard Allemandou}

\section{(2) OpenEdition}

Journals

Édition électronique

URL : https://journals.openedition.org/tc/8817

DOI : $10.4000 /$ tc. 8817

ISSN : 1952-420X

Éditeur

Éditions de l'EHESS

\section{Édition imprimée}

Date de publication : 17 mai 2018

Pagination : 152-167

ISBN : 978-2-7132-2750-9

ISSN : 0248-6016

Référence électronique

Gérard Allemandou, "Le stockfisch et la morue mis en recette », Techniques \& Culture [En ligne], 69 |

2018, mis en ligne le 17 mai 2020, consulté le 29 septembre 2022. URL: http://

journals.openedition.org/tc/8817; DOI : https://doi.org/10.4000/tc.8817 


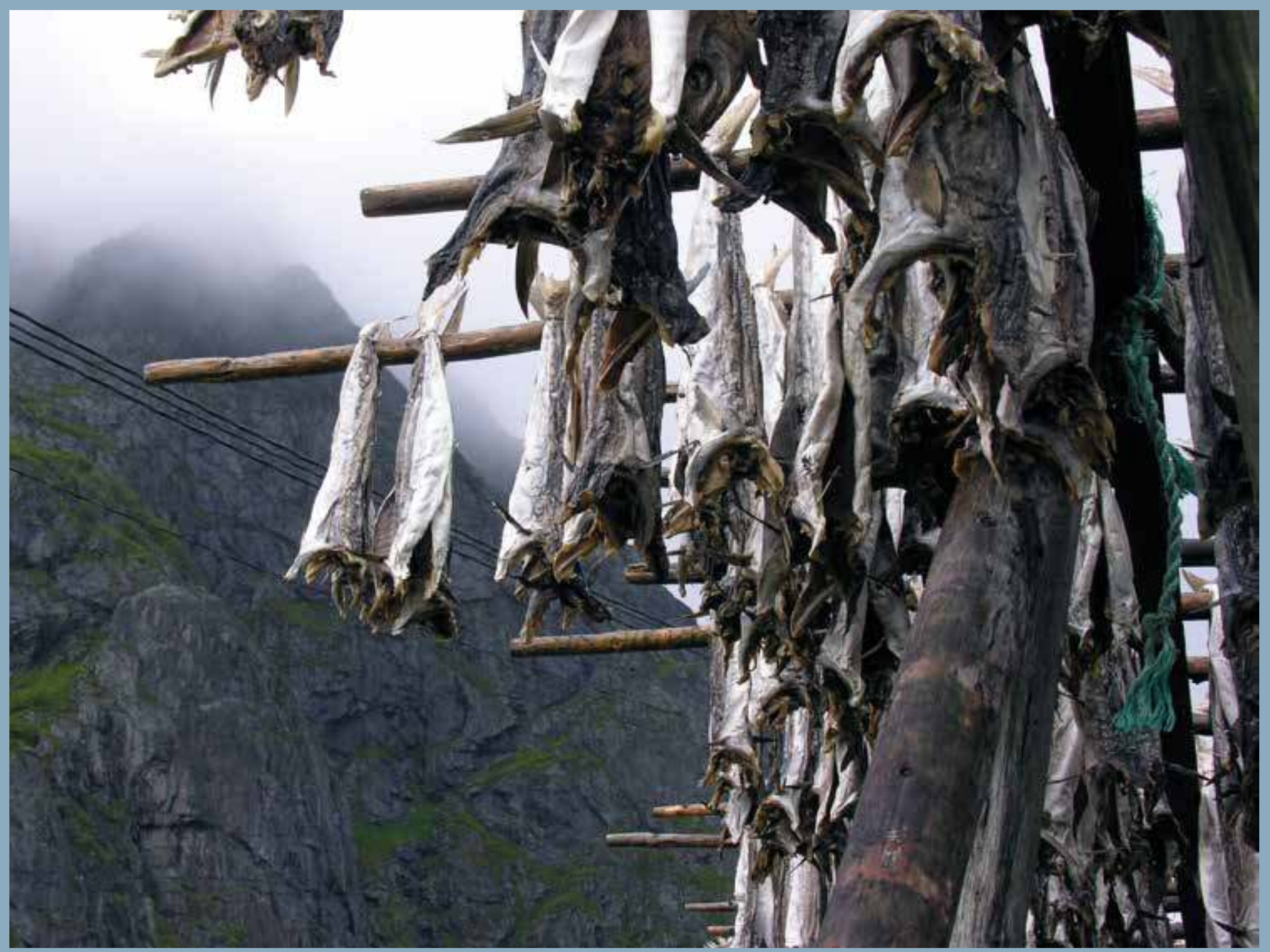




\section{Le stockfisch et la morue mis en recette}

\section{Manger du poisson sur tout le territoire français depuis le Moyen Âge}

Le poisson, source de protéine animale, est, grâce aux différents modes de conservation dont il est l'objet (séchage, salage, conservation dans de l'huile ou de la saumure), un produit alimentaire largement commercé depuis le haut Moyen Âge. À partir du milieu du xvi ${ }^{e}$ siècle le cabillaud est salé ou salé et séché sous le nom de morue. Les différentes appellations de cette conserve variant en fonction des modes et des périodes de pêches, des techniques de conservation, des pays, nous permettent de parler de «morues» au pluriel, tant les produits sont nombreux.

Nous proposons ici d'étudier deux recettes, toujours usitées aujourd'hui, qui apparaissent à la fin du XIve siècle ${ }^{1}$ dans des ouvrages de cuisine francophones. Elles présentent toutes deux la particularité de proposer l'apport de « gras » : huile, graisse de porc ou d'oie, voire beurre ou crème, pour assouplir et faciliter la préparation du poisson sec en effiloché. Depuis le Moyen Âge, le poisson n'est pas considéré comme de la «chair» et est jugé «ne pas tenir au corps». Lajout de gras lui redonne la forme d'un plat consistant compensant ainsi le traitement subi pour sa conservation.

À travers leurs histoires, nous mettons en évidence les liens qui existent entre techniques de conservation et modalités de consommation.

\section{Le premier cabillaud conservé: le stockfisch}

Le stockfisch est une conserve de cabillaud séché mais non salé. Les pays du Nord de l'Europe, d'où cette préparation est issue, ne disposent en effet que de sel gemme qui ne permet pas une conservation satisfaisante (Knockzert 2002 : 29). D'autre part la chair blanche et maigre de ces poissons ronds ne supporte pas le fumage. La première et seule occurrence dans les ouvrages de 
1. Le stockfisch est produit exclusivement en Norvège où les conditions climatiques permettent ce mode de conservation délicat à réaliser sans sel. Le poisson aussitôt pêché est saigné, étêté, lavé à l'eau de mer, puis mis à sécher par deux, attachés par la queue en «une poignée» (Duhamel du Monceau 1772: 71), avant d'être disposé en hauteur sur des perches en bois.

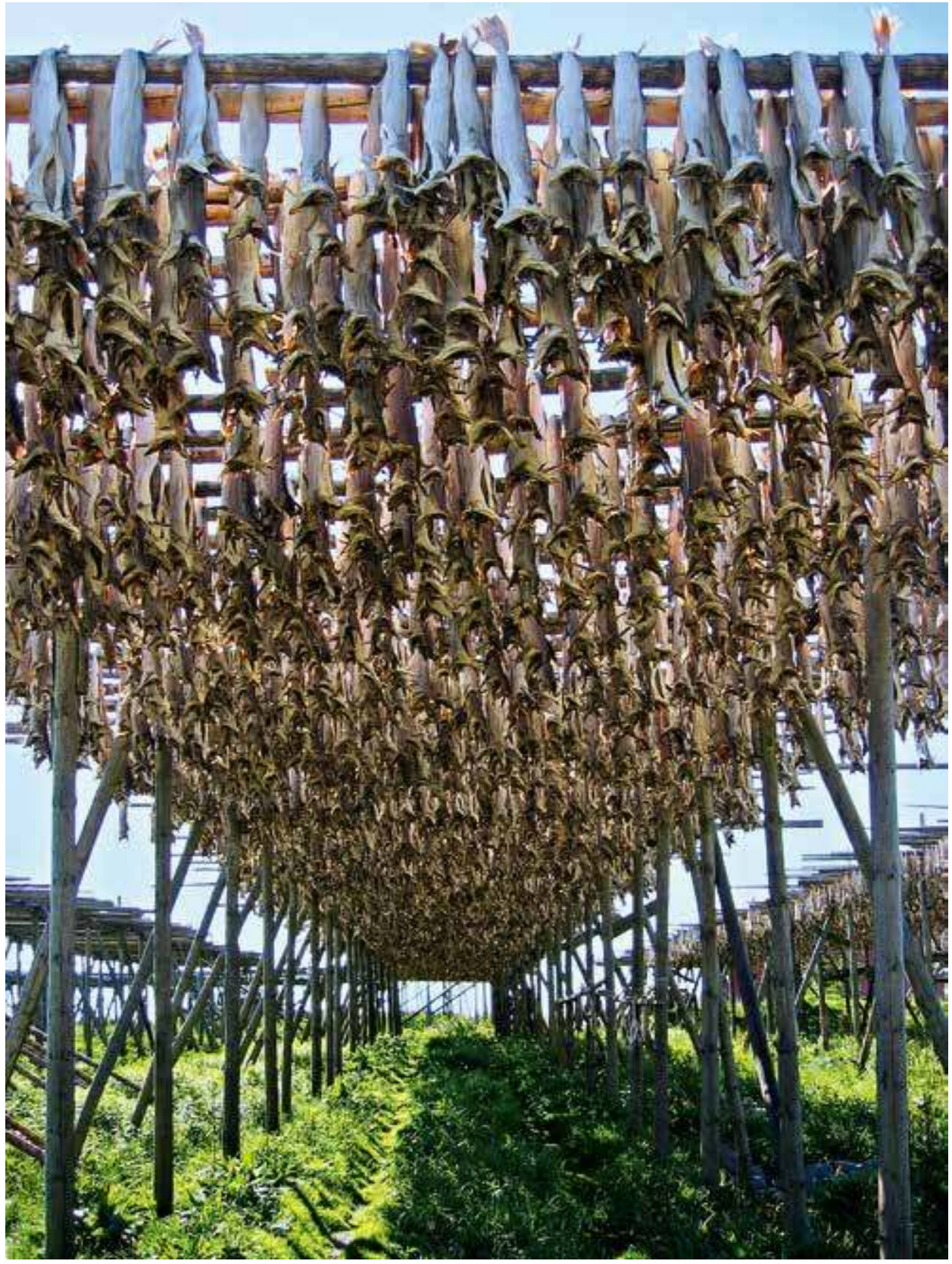


cuisine se trouve dans Le Ménagier de Paris (1992 [1393] : 195). Du Moyen Âge au Xvile siècle, ce stockfisch devient le viatique du voyageur qui le met dans sa besace, l'ordinaire des institutions (Hémardinquer 1960: 85-92), des armées en campagne et du marin qui l'emporte en mer. Pour les congrégations religieuses et les orphelinats, il améliore l'ordinaire des cent quarante jours de maigre imposés par l'Église et reste la ressource des garde-manger vides, du moins pour les plus nantis car le produit reste à cette période relativement coûteux (Couperie 1964: 468). Son commerce est le fait, au Xvile siècle, des navires hollandais, danois et anglais qui en ont le monopole. Ils approvisionnent l'Espagne, le Portugal, la Provence par Marseille (Delamare 1729) et desservent l'Italie par Gênes et Venise avant de l'exporter vers le Levant.

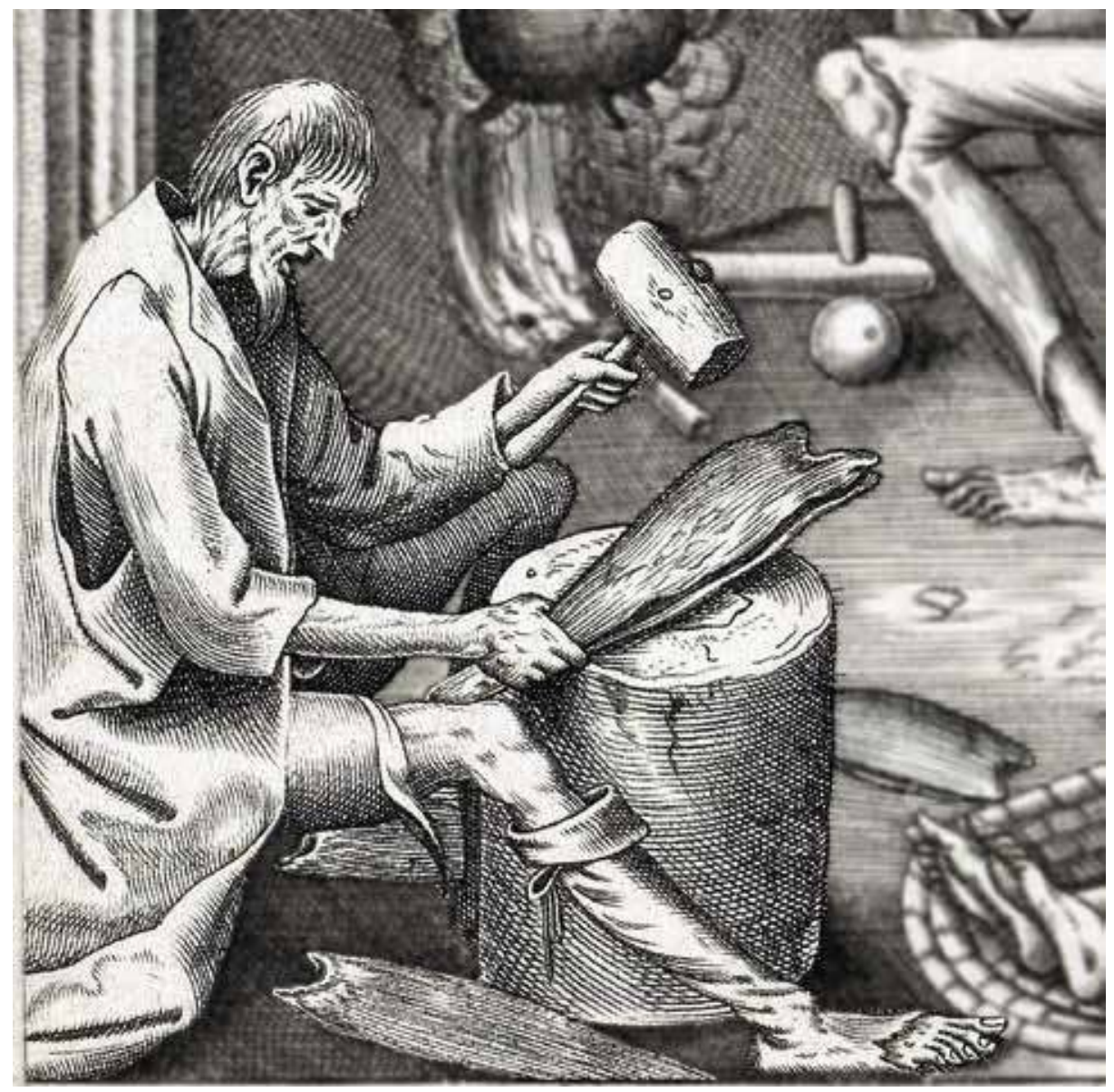

2. D'après un détail. Gravure inspirée de Pieter Brueghel dit l'Ancien, 1563 


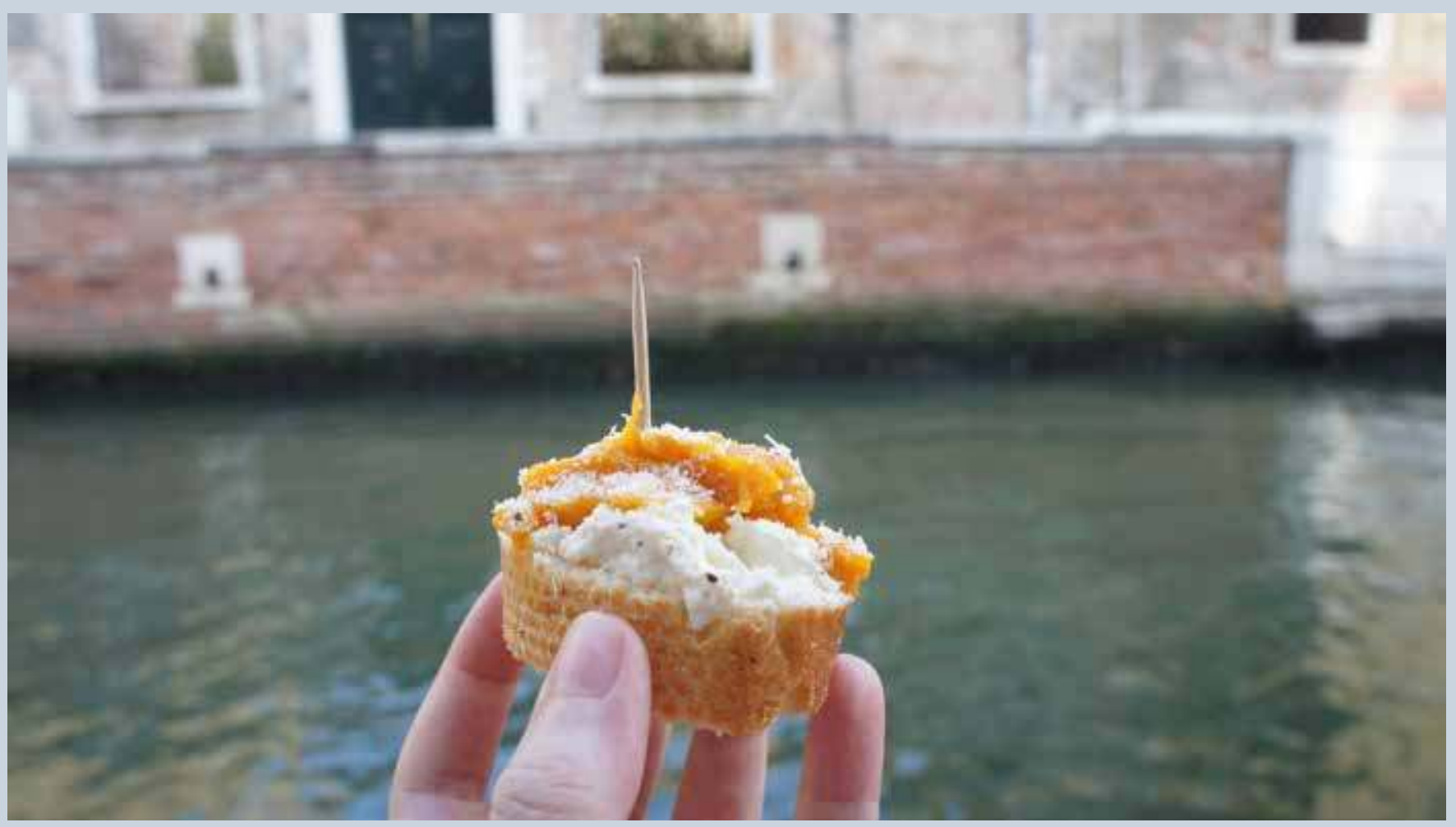

3. L'Italie reste aujourd'hui encore le second importateur mondial derrière le Nigeria, avec 15000 tonnes annuelles. A Venise, il existe une recette, très populaire, pratiquement identique à la brandade de Provence: «baccalà mantecato », servie ici sous forme de cicchetti, nom donné aux tapas vénitiennes. La Cantinone Via Schiavi 


\section{I'estofi, une survivance surprenante}

En France, une recette de stockfisch, l'estofi, est toujours consommée régulièrement entre Rodez et Cahors, autour de Decazeville, Figeac et Villefranche-de-Rouergue, jusqu'au Ségala de Carmaux. Dès le début du xvire siècle (Mergoil 1989: 37), le poisson arrivait par la Dordogne et le Lot dans les gabarres qui remontaient depuis Bordeaux jusqu'à Livinhac le Haut avec le sel, les épices et des produits exotiques. Comment expliquer sa présence aujourd'hui encore dans une aire aussi restreinte que celle du Rouergue et du Quercy, et aussi éloignée de la mer? Les historiens (Mergoil op. cit.) n'ont pas tranché et proposent plusieurs origines hypothétiques: les pèlerins de Saint-Jacques ou des soldats, ceux d'Édouard III ou de Louis XIV revenant des Flandres?

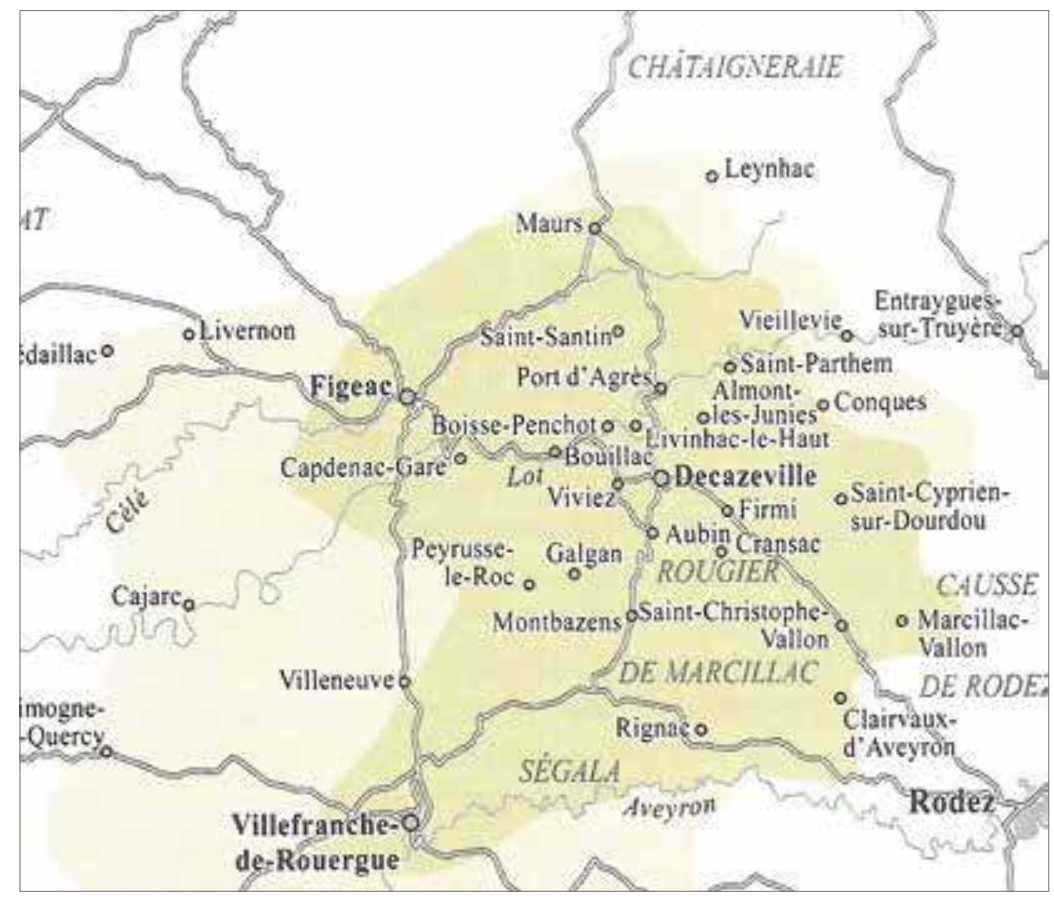

La carte de la consommation d'estofi en Rouergue et Quercy

L'estofinade en Rouergue et Quercy (d'après Guy Mergoil 1989:34) in Bernad \& Crozes 2012.

Lors de l'exode rural du xixe siècle, les paysans locaux qui se tournaient vers la mine pour assurer le pain quotidien et quittaient leurs villages emportèrent leurs pratiques alimentaires. L'estofi, déjà ancré dans l'habitude des repas pris en commun, fut conservé dans ce nouveau contexte ouvrier. On peut raisonnablement penser qu'il a pu contribuer à la formation d'une mémoire partagée supportée par le maintien de la préparation de ce plat, notamment au travers des repas collectifs, appelés « estofinade ». L'estofi était préparé lors des soirées organisées 
pour peler les châtaignes, les veilles de fêtes et surtout, dans cette région viticole, avant que le phylloxéra ne détruise le vignoble, pour les fins de vendanges. Après 1780, date de l'arrivée de la pomme de terre en Segala (Mergoil: 37), la recette évolue d'une préparation de morue effeuillée, liée au gras, certainement mangée sur du pain, à sa forme actuelle, qui s'installe au début du Xix siècle. Aujourd'hui, il faut l'occasion d'une réunion de famille - le repas du Vendredi saint, par exemple, jour maigre -, ou la veille des cousinades ${ }^{2}$, pour qu'elle occupe l'ordinaire, n'étant pas jugée assez noble pour les repas festifs. Elle reste bien présente cependant dans les repas collectifs des associations, le partage de l'après match des rugbymans et, depuis le retour de la viticulture, à nouveau celui de fin de vendange. Une Confrérie de l'estofi se charge même d'actualiser ce savoir-faire, pratiqué par une douzaine de restaurateurs, les «maîtres-estofinaïres ».

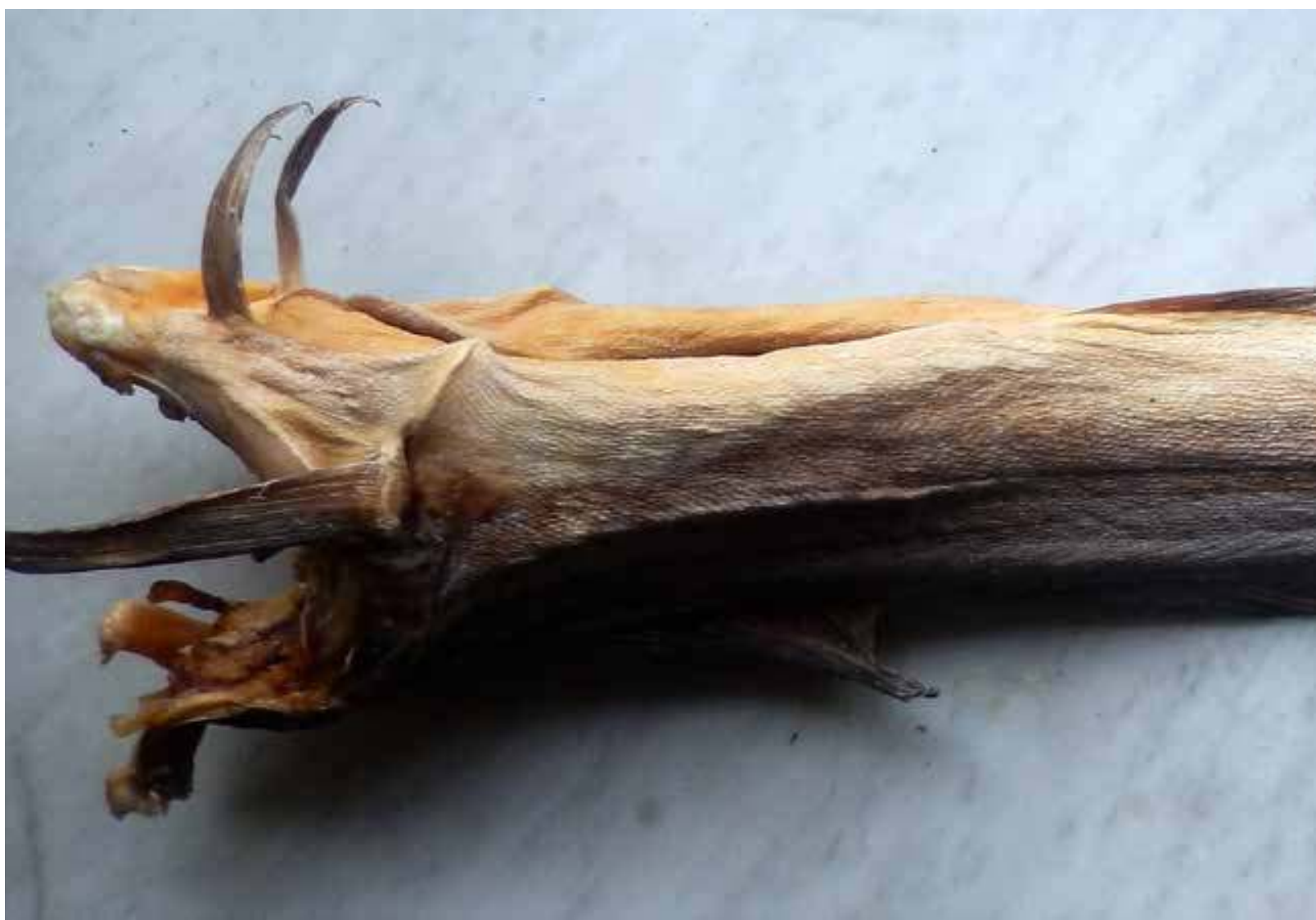


4. Un poisson entier d'environ $1,35 \mathrm{~kg}$ convient pour 14 convives. Sa réhydratation peut prendre une semaine, ce qui nécessite de changer l'eau chaque jour. Cuit, sans bouillir, environ 30 minutes, il sera paré: peau et arêtes ôtées, et mis «en feuille». On y ajoute $2,5 \mathrm{~kg}$ de pommes de terre qui seront cuites dans l'eau du poisson, puis égouttées et grossièrement écrasées au presse-purée manuel. 


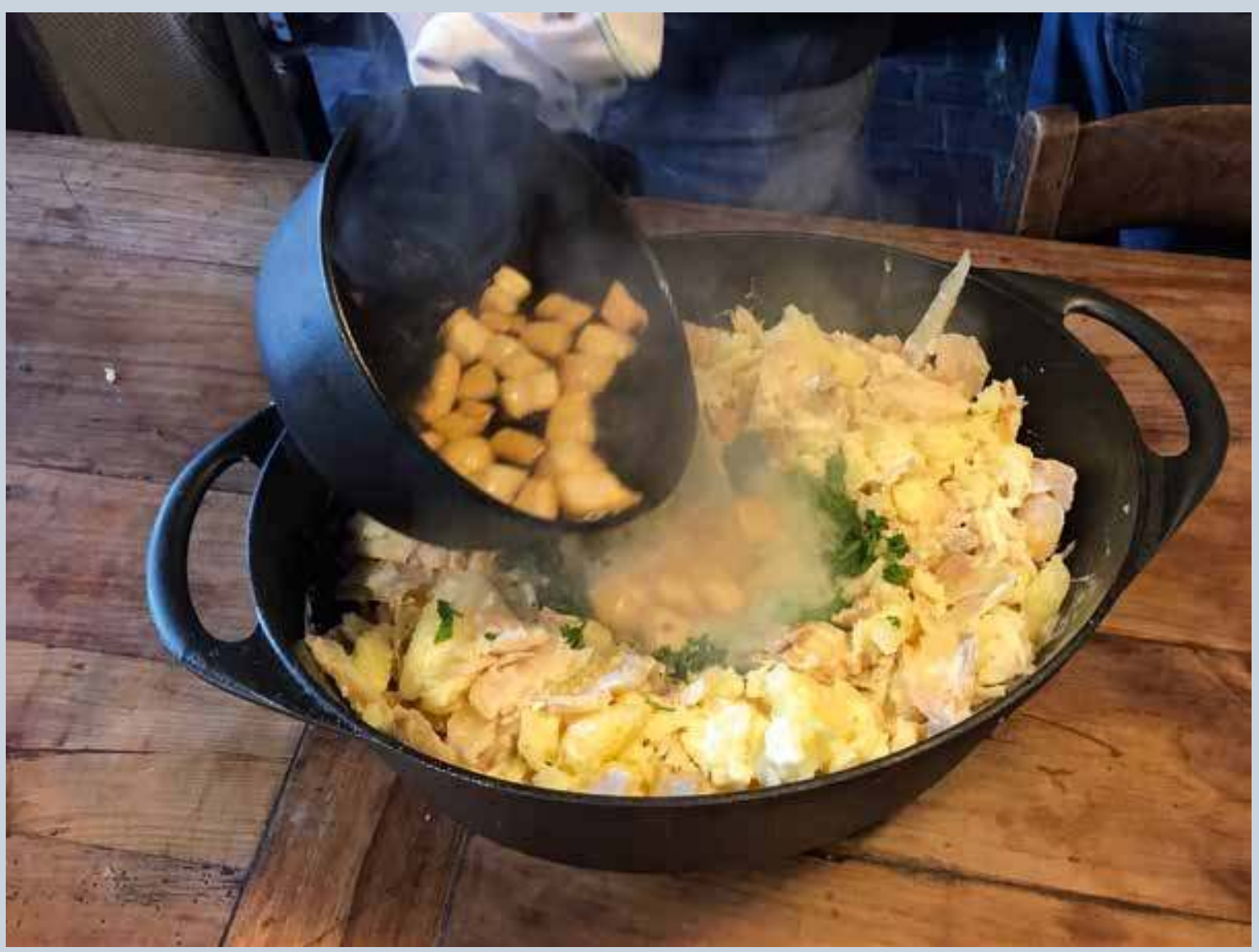

5. Une fois le poisson rajouté, un puits est formé pour mettre un bouquet de persil et $30 \mathrm{~g}$ d'ail ciselés, quatre œufs entiers et $400 \mathrm{~g}$ de lard coupé en lardons frits dans $30 \mathrm{cl}$ d'huile neutre. Après avoir mélangé à la cuillère en bois, la préparation est achevée avec l'ajout de $300 \mathrm{~g}$ de crème fraîche. 


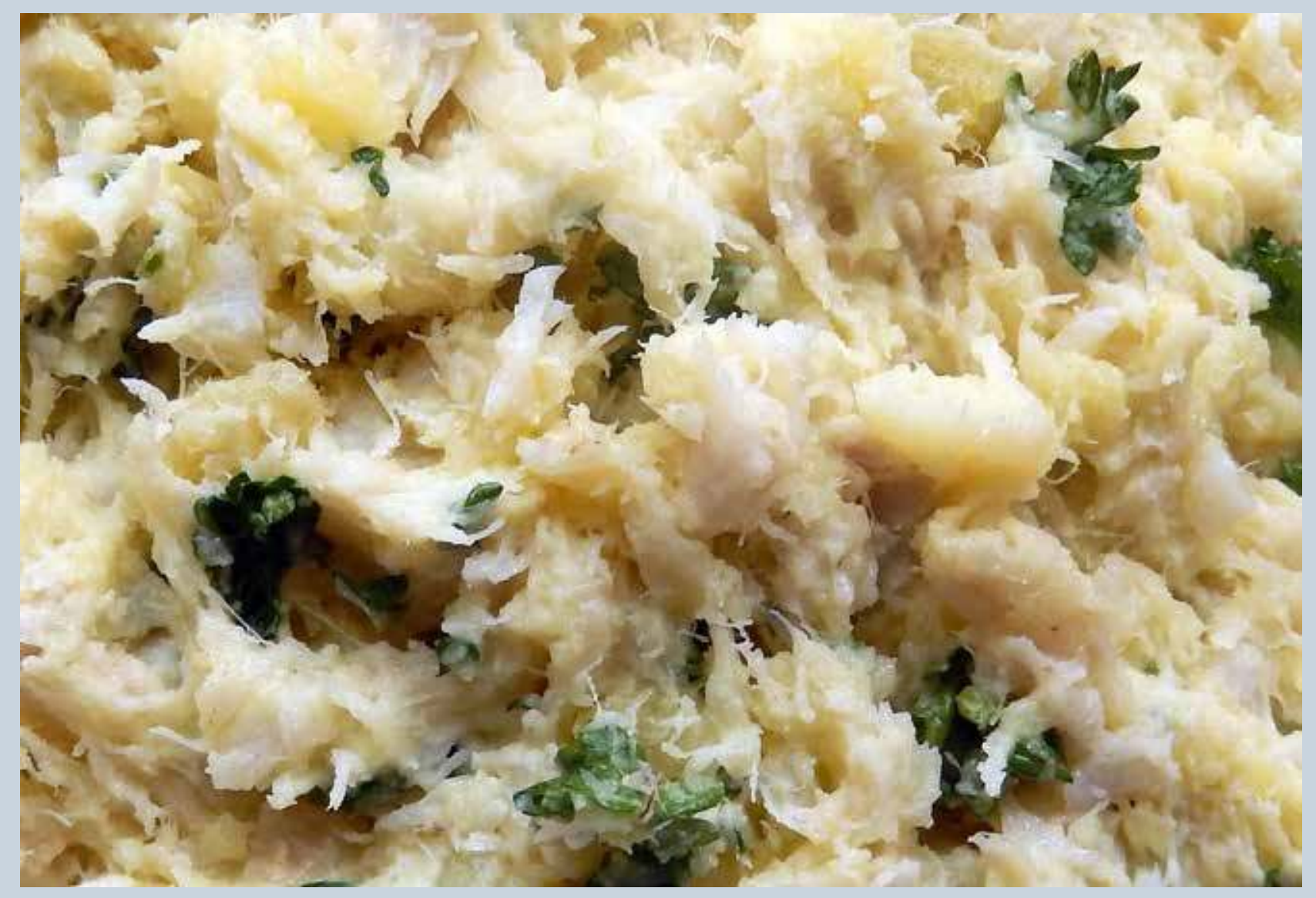

6. Salé et poivré, l'estofi est servi dans un saladier, quand la marmite n'est pas mise directement sur la table. Il est jaune clair parsemé de vert. Selon l'usage, il doit être souple sans être trop gras, moelleux et surtout pas sec. 


\section{La brandade : recette historique}

Les premiers navires français, au milieu du xvie siècle, s'armèrent pour aller à la «grande pêche» vers Terre-Neuve et rapporter le cabillaud salé, différent du stockfisch exclusivement séché. Ce cabillaud salé est nommé morue verte et s'il est également séché: morue ou merluche. Progressivement, cette conserve française a pris la place du stockfisch norvégien. Sous différentes formes elle va gagner toute la France depuis les différents ports où elle débarque. Le tableau page 164 recense, en fonction des lieux, des types, des saisons de pêches, des formes d'armements, des méthodes de conservations, les destinations portuaires où la morue est livrée et d'où elle continuera son chemin par terre ou par mer pour atteindre tout le territoire (Delamare 1729, Duhamel Dumonceau 1772).

Beaucoup des ports de l'Atlantique armèrent pour la «grande pêche» et, au retour, certains bateaux, ceux de Saint-Malo surtout, en faisaient commerce, gagnant directement le Sud-Est pour approvisionner Marseille de morue salée et séchée (Vié 2001). La «verte» supportant

7. La morue est livrée en caisse de $5 \mathrm{~kg}$. Pour la brandade parmentière, une «morue verte» de cabillaud norvégien, donc salée et non séchée, est utilisée ici. Les filets sont mis à dessaler dans l'eau froide pour 48 heures. Pour 12 convives $2,52 \mathrm{~kg} d e$ morue sont mis à cuire dans un rondeau à feu doux, eau fumante.

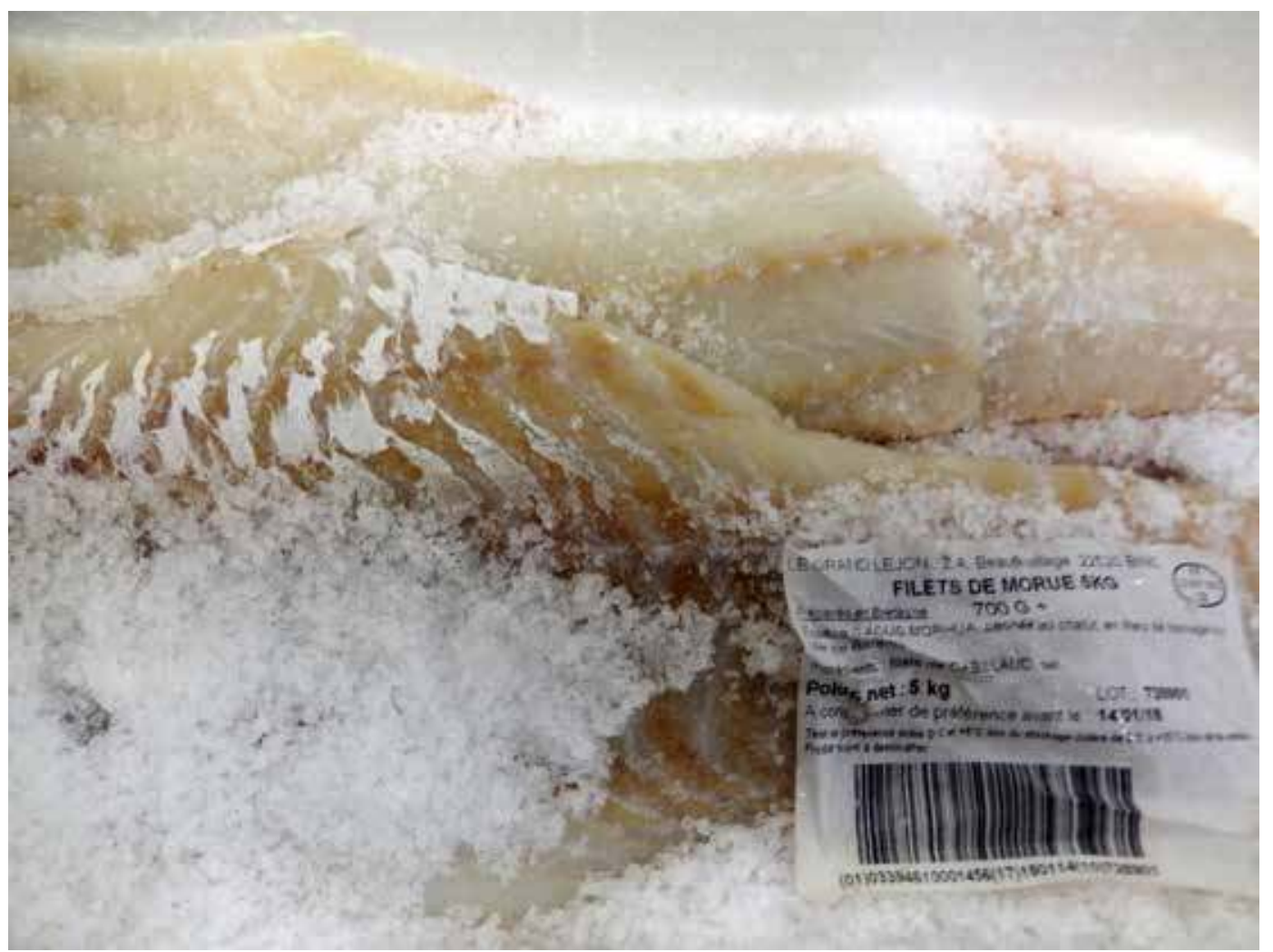


difficilement les conditions climatiques régionales (Duhamel Dumonceau 1772), la morue plus rapide à mettre en œuvre, moins sèche, et surtout d'un goût jugé bien plus agréable pour l'époque car moins fort, prit la place du stockfisch. La brandade s'inscrit alors dans le patrimoine culinaire du Sud-Est, en Provence comme en Languedoc. Les Nîmois prétendent que le cuisinier Charles Durant l'aurait créée à Alès, dans les années 1790. Pourtant, la plus ancienne des recettes date, à notre connaissance, de 1767. Dans une lettre au Journal Economique, un lecteur donne la recette: «Façon de la préparer en usage à Marseille». D'autres articles de ce journal expliquent que la morue est alors un élément essentiel de l'alimentation populaire.

À Paris, cette recette a fait la renommée du restaurant Les Frères Provençaux, installé au Palais Royal en 1786, qui bâtit sa réputation grâce à Grimod de la Reynière. Il cita le restaurant pour sa brandade dans ses Almanachs et en divulgua même la recette (Grimod de la Reynière 1805: 201-207). Car les recettes de brandade se trouvent le plus souvent ailleurs que dans les livres de cuisine, comme Journal, Dictionnaire, Manuel de conversation, etc. Il semble que les cuisiniers de renom, ceux qui publient, ont oublié cette recette pour la cantonner au populaire. Vers 1815, Durant, qui officie dans différents restaurants, mettra la brandade à disposition des consommateurs comme plat de traiteur. Ainsi, à sa suite, hôtels, restaurants et traiteurs offrent de la brandade à emporter. La recette, qui paraît simple, a le défaut d'être longue à mettre en œuvre et demande un certain tour de main. Bien que citée dans sa forme initiale dans la littérature de cuisine (Escoffier 1903, Montagné 1938), elle s'éloigne peu à peu de la «haute cuisine», ne se maintenant que dans les familles et la restauration collective. Ainsi, dans La morue du Comité de propagande pour la consommation de morue, la recette de brandade est suivie d'une «Brandade de morue en quenelles» destinée aux collectivités et qui contient de la pomme de terre (Collectif 1951 : 32-33). Ce moindre coût favorise certainement l'inscription de la brandade dite "parmentière» dans la cuisine collective et elle sera largement mise en rayon dans les supermarchés. Elle reste, en tant que plat préparé, en France, la morue la plus consommée, en dehors des familles immigrées portugaises ou italiennes. Les deux recettes présentées ici, proches l'une de l'autre, tiennent du même principe, le mélange de morue émiettée avec du gras, les protéines hypotenseuses favorisant en chauffant la liaison de l'ensemble en une pâte. La pomme de terre vient densifier le plat en l'assouplissant, et ce, pour un faible coût. 


\begin{tabular}{|c|c|c|c|c|c|c|}
\hline & \multicolumn{3}{|c|}{ américaine } & \multicolumn{3}{|c|}{ europeenne } \\
\hline lieu de piche & \multicolumn{2}{|c|}{ Atlantique Nord, Terre Neuve } & Atlantique Nord, Terre Neuve & \multicolumn{2}{|c|}{ mer du Nord, Dogger - Bank, Islande } & $\begin{array}{c}\text { Scandinavie, Baltique, } \\
\text { Norvège }\end{array}$ \\
\hline $\begin{array}{l}\text { periode de } \\
\text { peche }\end{array}$ & \multicolumn{2}{|c|}{ mai a septembre } & avril a septembre & \multicolumn{2}{|c|}{ fevrier-mars / juillet-sept } & $\begin{array}{c}\text { préparation de janvier a fin } \\
\text { avril }\end{array}$ \\
\hline $\begin{array}{l}\text { ptriode de } \\
\text { livraison }\end{array}$ & \multicolumn{2}{|c|}{ arrive de septembre a decembre } & arrive d'octobre a decembre & \multicolumn{2}{|c|}{ Ia * morue nouvelle: } & permanente \\
\hline port de départ & \multicolumn{2}{|c|}{ les ports du * Ponant $*$, Atlantique et Manche } & $\begin{array}{c}\text { Normandie, Bretagne, Poitou, } \\
\text { Basques }\end{array}$ & uniquement Dunkerque & Norvegien et Hollandais & $\begin{array}{l}\text { Bergen puis les ports } \\
\text { hanseatiques }\end{array}$ \\
\hline type du produit & \multicolumn{2}{|c|}{ morue salte verte ou dite blanche } & $\begin{array}{l}\text { morue salée et stechee ou } \\
\text { merluche }\end{array}$ & \multicolumn{2}{|c|}{ morue salee verte ou dite blanche } & morue stchee \\
\hline $\begin{array}{l}\text { dinomination } \\
\text { usuelle }\end{array}$ & $\begin{array}{l}\text { " morve ronde } n \text { dite } \mathrm{S} \text { la } \\
\text { françalse, en grenier }\end{array}$ & $\begin{array}{l}\text { a morue plate s dite a } \\
\text { Thollandaise en banl }\end{array}$ & merluche: & \multicolumn{2}{|c|}{$\begin{array}{l}\text { morue plate en tonneau dite d'Aberdham ou } \\
\text { d'islande }\end{array}$} & stockfisch ou baton \\
\hline $\begin{array}{l}\text { technique de } \\
\text { dtcoupe }\end{array}$ & $\begin{array}{l}\text { Ouverte jusqu'au cloaque en } \\
\text { laissant une arte vers la } \\
\text { queve, salte, mise en cale }\end{array}$ & $\begin{array}{l}\text { Ouverte à plat, désossée } \\
\text { entièrement, salée, mise en } \\
\text { cale }\end{array}$ & $\begin{array}{l}\text { ouverte } 3 \text { plat, désossee, } \\
\text { salée, mise en tas sur le } \\
\text { bateau, le échafaud } \%\end{array}$ & $\begin{array}{l}\text { découpée en filet, } \\
\text { tgoutte, salte }\end{array}$ & $\begin{array}{c}\text { découpte en deux avec ou } \\
\text { sans the }\end{array}$ & simplement tetétée et vidte. \\
\hline \multirow{2}{*}{$\begin{array}{l}\text { technique de } \\
\text { salage ow } \\
\text { stchage }\end{array}$} & $\begin{array}{l}\text { après sechage, nouveau } \\
\text { salage et installation sur des } \\
\text { nattes de joncs, en pile } \\
\text { jusqu'au plafond }\end{array}$ & $\begin{array}{c}\text { paqué en tonneau avec du } \\
\text { sel }\end{array}$ & $\begin{array}{c}\text { lavte, tgouttee, étendue sur } \\
\text { une grève ou sur des rochers, } \\
\text { empiltes en tas au soleil } \\
\text { pendant environ sa } 6 \text { mois }\end{array}$ & $\begin{array}{c}\text { salee au sel du } \\
\text { Portugal, en couche en } \\
\text { tonneau }\end{array}$ & $\begin{array}{l}\text { salee au sel du Portugal, } \\
\text { en couche en tonneau }\end{array}$ & \multirow{2}{*}{$\begin{array}{l}\text { mise a secher a lair froid } \\
\text { et sec du nord par deux } \\
\text { attachées par la queve }\end{array}$} \\
\hline & $\begin{array}{c}\text { pas de barrique ni de pressage, } \\
\text { mises par deux avec une } \\
\text { Scelle, } \propto \text { la poignee } \%\end{array}$ & $\begin{array}{l}\text { de reteur au port, lavee, } \\
\text { egouttée, parte, mise en } \\
\text { barrique, salke à sel sec, } \\
\text { pressee, } 25 / 30 \text { poissons, } \\
10 / 15 \mathrm{~kg}\end{array}$ & $\begin{array}{l}\text { selon le séchage morue } \\
\text { blanche ou noire ou pinte }\end{array}$ & $\begin{array}{l}\text { lavte, egoutté, parte, } \\
\text { mise en barrique, salee } \\
\text { a sel sex, presste }\end{array}$ & $\begin{array}{l}\text { lavee, egouttée, parée, } \\
\text { mise en barrique, salée a } \\
\text { sel sec, pressete }\end{array}$ & \\
\hline $\begin{array}{l}\text { lieu de } \\
\text { consommation }\end{array}$ & $\begin{array}{l}\text { jugée de qualité dans toutes } \\
\text { les metropoles de bords de } \\
\text { mer et Paris ; la morue } \\
\text { nouvelle est particulièrement } \\
\text { appreteile }\end{array}$ & $\begin{array}{l}\text { dans toutes les métropoles } \\
\text { de bords de mer et Paris. } \\
\text { partiellement exportée }\end{array}$ & $\begin{array}{l}\text { en provenance de la } \\
\text { « Nouvelle Angleterre }{ }^{\text {, }} \\
\text { Iivres aux Antilles, aussi par } \\
\text { le commerce triangulaire } \\
\text { depuis Nantes et St Malo }\end{array}$ & $\begin{array}{l}\text { Dunkerque, de } \\
\text { meilleure qualité }\end{array}$ & $\begin{array}{l}\text { XVlie-Angleterre, } \\
\text { Hollande / XVille- } \\
\text { Hollande, Norvège, } \\
\text { Dunkerque }\end{array}$ & $\begin{array}{c}\text { commerce par les Danois, } \\
\text { les Allemands, les } \\
\text { Norvigiens } \\
\text { essentiellement vers le } \\
\text { sud }\end{array}$ \\
\hline $\begin{array}{l}\text { transit en } \\
\text { France }\end{array}$ & $\begin{array}{c}\text { Dieppe pour Paris, Le Havre, } \\
\text { Honfleur, Olonne, Nantes, La } \\
\text { Rochelle, Bordeaux puis par } \\
\text { voie d'eau vers l'intérieur ou } \\
\text { par roulage }\end{array}$ & $\begin{array}{c}\text { Nantes, Orleans par fleuve, } \\
\text { Paris par roulage }\end{array}$ & $\begin{array}{c}\text { Bordeaux, Bayonne puis les } \\
\text { fleuves, Marseille en octobre } \\
\text { pour repartir vers l'Espagne, } \\
\text { l'Italie jusqu'en Sicile, Malte, } \\
\text { le Levant }\end{array}$ & Le Havre puis la Seine & Le Havre puis la Seine & $\begin{array}{l}\text { Marseille, Stete, Bordeaux } \\
\text { Bayonne puis les fleuves, } \\
\text { l'Italie vers le Levant. }\end{array}$ \\
\hline
\end{tabular}

Ces deux courts récits illustrent comment la conserve du cabillaud, stockfisch ou morue, a été un élément important de l'alimentation française en permettant de manger du poisson pratiquement toute l'année depuis le xvie siècle sur l'ensemble du territoire et dans différents milieux sociaux. Aujourd'hui, leur utilisation perdure dans deux recettes du quotidien. L'une est pratiquée dans les repas collectifs populaires alors que l'autre s'est éloignée de la cuisine ménagère, comme de celle des restaurants, pour devenir un plat préparé mis à disposition par la distribution ou servi dans la cuisine de collectivité. Ces commensalités dissemblables mettent en évidence combien les liens entre techniques de conservation et pratiques de consommation peuvent prendre des formes différentes, l'équilibre entre ces deux termes variant dans le temps long de l'histoire alimentaire. 
8. La morue cuite est égouttée et effeuillée pour enlever les peaux et les arêtes. 1,150 kg de pommes de terre bintje cuites, égouttées, encore fumantes, sont écrasées au presse-purée manuel. Elles sont ensuite incorporées à la morue avec $25 \mathrm{~g}$ d'ail ciselé, $400 \mathrm{~g}$ de crème fraîche et $250 \mathrm{~g}$ d'huile d'olive.

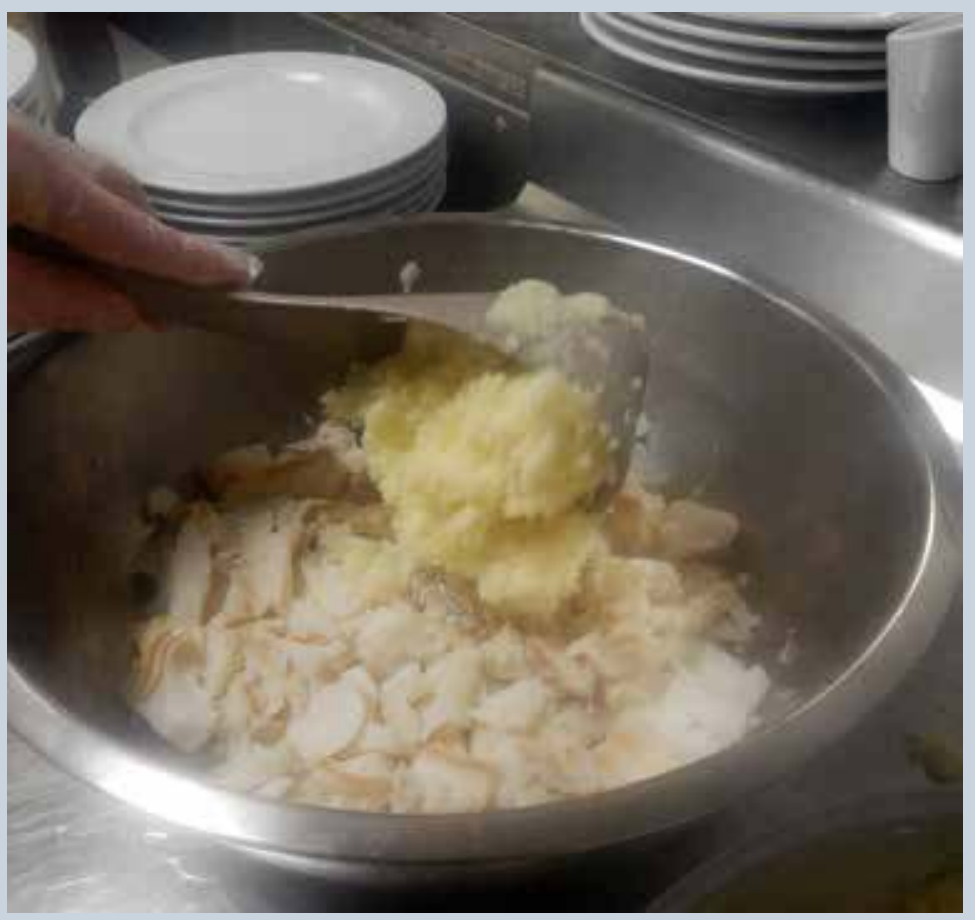

9. La préparation est travaillée à la main pour que le mélange devienne homogène. Elle est goûtée pour ajuster le sel. 


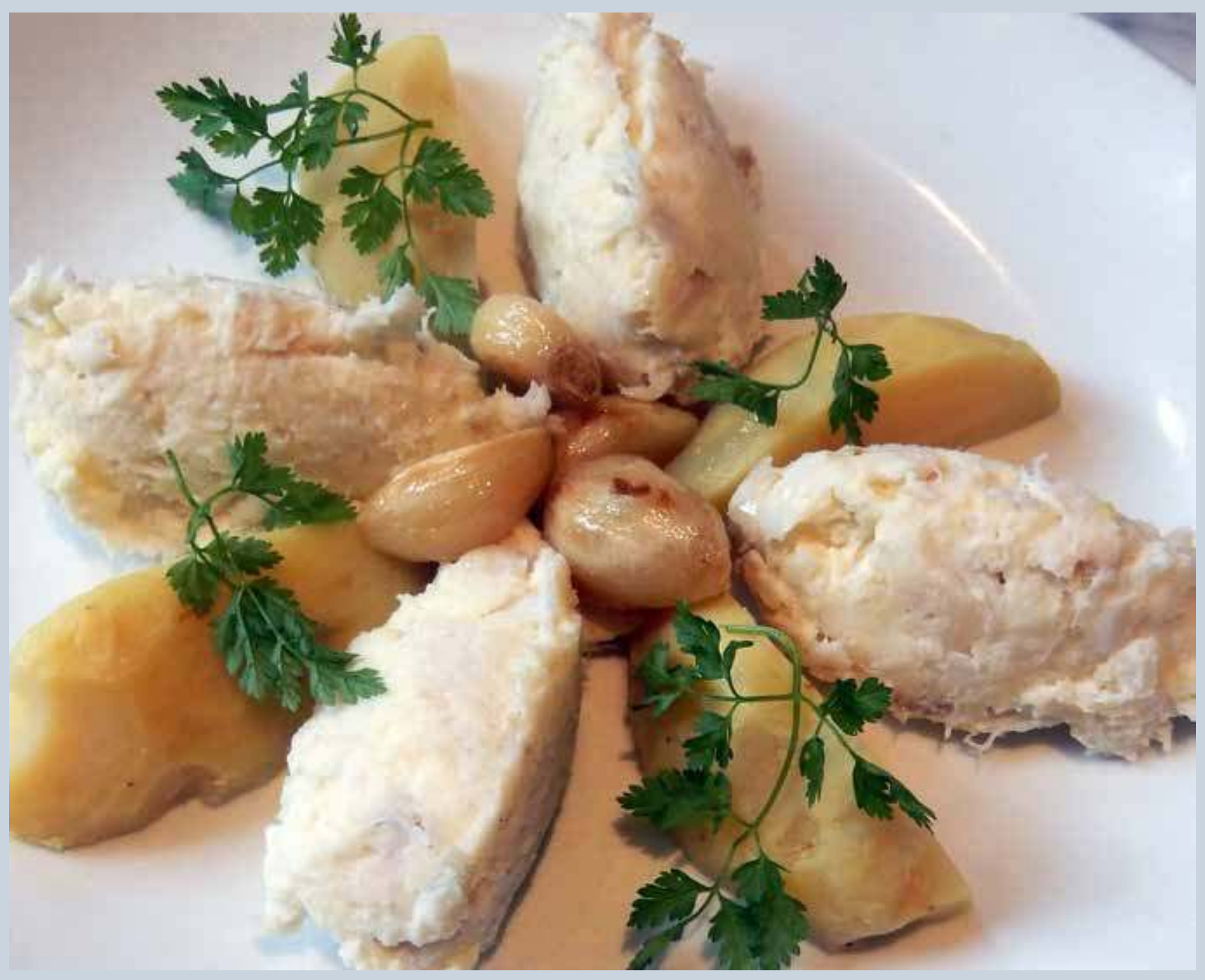

10. L'assiette de «brandade rustique» servie ici au restaurant la Cagouille (Paris, où l'auteur a officié pendant plus de 30 ans) : quenelles de brandade, quarts de pomme de terre, ail rôti au four et pluches de cerfeuil, un trait d'huile d'olive. 


\section{Notes}

1. La morue salée est mentionnée en 1392 dans Le Viandier de Taillevent, et le stockfish dans Le Ménagier de Paris de 1393. Le cuisinier François de La Varenne propose une recette de morue en 1651, puis des recettes sont présentes dans les textes jusqu'au XIX ${ }^{e}$ siècle alors que le stockfich disparaît.

2. Réunions de famille qui dure plusieurs jours.

\section{I'auteur}

Gérard Allemandou est cuisinier-restaurateur, créateur du restaurant la Cagouille à Paris, spécialiste de la cuisine du poisson, il dirige la revue Papilles de l'association des Bibliothèques gourmandes et explore l'histoire de la cuisine, en particulier celle des produits de mer et de rivière.

\section{Iconographie}

Image d'ouverture. Stockfish, Les îles Lofoten (Norvège). CC by Paolo Tonon.

1. CC by Ellen van Bogedom.

2. CC by The Rijksmuseum. Gravure réalisée par Peter van der Heyden, La cuisine maigre, 1563, où un « démuni » frappe un stockfisch avec un maillet en bois.

\section{Références}

Bernad, C. \& D. Crozes 2012 L'estofi. Arles: Le Rouergue. Collectif 1951 La morue. Paris: Comité de propagande pour la consommation de la morue.

Couperie, P. 1964 «L'alimentation au Xvil ${ }^{e}$ siècle : les marchés de pourvoierie », Annales. Économies, Sociétés, Civilisations 3 (19): 467-479. DOI: 10.3406/ ahess.1964.421170.

Delamare, M. 1729 Traité de police. Amsterdam : Aux dépens de la Compagnie.

Duhamel du Monceau, H.-L. 1772 Traité des pesches, et histoire des poissons qu'elles fournissent, tant pour la subsistance des hommes que pour plusieurs autres usages qui ont rapport aux arts et au commerce. Paris: Saillant $\&$ Nyon / Veuve Desaint Librairies.

Escoffier, A. 1903 Le guide culinaire. Paris: L'art culinaire.
3. Tous droits réservés: (c) Marion Bothorel.

Crédits pour l'ensemble des images à l'exception des précédentes. (C) Gérard Allemandou.

Grimod de la Reynière, A. B. L. 1805 L'Almanach des Gourmands. Paris: Maradan.

Hémardinquer, J. J. dir. 1960 «Pour une histoire de l'alimentation», Cahiers des Annales 28.

Journal Economique 1767 Paris: Chez Antoine Boudet.

Knockzert, C. 2002 Le fumage du poisson. Paris: Ifremer.

Le Ménagier de Paris 1992 Traité de morale et d'économie domestique composé vers 1393 par un bourgeois parisien. Lille: Régis Lehoucq.

Mergoil, G. 1989 «Un îlot insolite de consommation du stockfisch: les confins Rouergue-Quercy » in J. Peltre $\& C l$. Thouvenot dir. Alimentation et Régions: actes du colloque «Cuisines, régimes alimentaires, espaces régionaux », Nancy: Presses Universitaires de Nancy.

Montagné, P. 1938 Larousse Gastronomique. Paris: Larousse. Vié, B. 2001 La morue. Paris: Jean-Paul Rocher.

\section{Pour citer l'article}

Allemandou, G. 2018 «Le stockfisch et la morue mis en recette. Manger du poisson sur tout le territoire français depuis le Moyen Âge», Techniques\&Culture 69 «Le temps des aliments», p. 152-167. 